\title{
A Theory of Brain Drain and Public Funding for Higher Education in
}

\author{
the U.S.
}

Felicia Ionescu

Linnea Polgreen*

\section{$1 \quad$ Technical Appendix}

This appendix provides proofs for all the results in our paper. We present and number them in the order they appear in the paper, by each section.

\section{Enrollment Under Heterogeneity in Public Funding}

Proposition 1.1. There is a threshold $\bar{a}$ such that all individuals with ability $a>\bar{a}$ will enroll in college, and all individuals with $a<\bar{a}$ will not go to college.

Proof. Wlog we restrict ourselves to the case where no one goes to college, $\mu_{0}=0$; there is an incentive for the most gifted to enroll, i.e. $g(0)-c>1$. Let $G_{1}(a)=g(0) a-c$, so $G_{1}(0)<$ $1<G_{1}(1)$. Then there exists $\bar{a}_{1}$ such that $G_{1}\left(\bar{a}_{1}\right)=1$ and $\mu_{1}=\int_{\bar{a}_{1}}^{1} f(z) d z=1-\bar{a}_{1}>\mu_{0}$. Let $G_{2}(a)=g\left(\mu_{1}\right) a-c$, so $G_{2}(0)<1<G_{2}(1)$. Then there exists $\bar{a}_{2}$ such that $G_{2}\left(\bar{a}_{2}\right)=1$ and $\mu_{2}=\int_{\bar{a}_{2}}^{1} f(z) d z=1-\bar{a}_{2}>\mu_{1}$. By induction, there exists $\bar{a}_{n}$ such that $G_{n}\left(\bar{a}_{n}\right)=1$ and $\mu_{n}=\int_{\bar{a}_{n}}^{1} f(z) d z=1-\bar{a}_{n}$ with $\left\{\mu_{n}\right\}$ an increasing, bounded sequence on $[0,1]$ and $\left\{\bar{a}_{n}\right\}$ a decreasing, bounded sequence on $[0,1]$. Then there exist $\mu$ and $\bar{a}$ such that $\lim \mu_{n}=\mu$ and $\lim \bar{a}_{n}=\bar{a}$ with $g(\bar{a}) \bar{a}-c=1$ and $\mu=1-\bar{a}$.

*Department of Economics, Colgate University, 13 Oak Drive, Hamilton, NY, 13346, email: fionescu@mail.colgate.edu; College of Pharmacy, University of Iowa, 115 S. Grand Avenue, Iowa City, Iowa 52242 email: linnea-polgreen@uiowa.edu. 
Proposition 1.2. Given that state 1 subsidizes education, regardless of the subsidy type, a higher fraction, $\mu_{1}$ will choose to go to college in state $1, \mu_{1}=\mu\left(\bar{a}_{1}\right)>\mu_{2}=\mu\left(\bar{a}_{2}\right)$. The fraction, $\mu_{1}$ increases with the subsidy.

Proof. By Proposition 1.1, there exist $\bar{a}_{1}$ and $\bar{a}_{2}$ such that all agents of type $a>\bar{a}_{1}$ go to college in state 1 , and all agents of type $a>\bar{a}_{2}$ go to college in state 2 . If state 1 gives a subsidy regardless of ability level, $G(a)=g(\mu(a)) a-c$ is the same as before. If state 1 chooses to condition the subsidy on ability level, the function is given by $G(a)=g(\mu(a)) a-c(a)$, which is continuous and strictly increasing in $a$ given $c^{\prime}(a)<0$, and $g(\mu)>g^{\prime}(\mu) a$. The two fractions that go to college are given by $\mu_{1}=\mu\left(\bar{a}_{1}\right)=\int_{\bar{a}_{1}}^{1} f(z) d z$ and $\mu_{2}=\mu\left(\bar{a}_{2}\right)=\int_{\bar{a}_{2}}^{1} f(z) d z$. Since $c_{1}<c$, it results that $g\left(\mu_{2}\right) \bar{a}_{2}-c_{1}>g\left(\mu_{2}\right) \bar{a}_{2}-c=1=g\left(\mu_{1}\right) \bar{a}_{1}-c_{1}$. This implies $\bar{a}_{1}<\bar{a}_{2}$, and the result follows.

\section{Brain drain}

\section{A The general problem}

Proposition 1.3. Given $\mu_{1}=\mu(\bar{a})$, the fraction who enrolls in college in state 1 , the fraction of agents who stay is $\lambda\left(\mu_{1}\right) \in\left[\frac{\mu_{1}}{2}, \mu_{1}\right]$ or $\lambda\left(\mu_{1}\right)=0$.

Proof. Suppose, by contradiction, that there is incomplete brain drain. Then $\lambda\left(\mu_{1}\right)<\mu_{1}-\lambda\left(\mu_{1}\right)$ and since $g$ is increasing it follows that $g\left(\lambda\left(\mu_{1}\right)\right)-g\left(\mu_{1}-\lambda\left(\mu_{1}\right)<0\right.$. Then for any $a, g\left(\lambda\left(\mu_{1}\right)\right) a-$ $g\left(\mu_{1}-\lambda\left(\mu_{1}\right)\right) a<0$. Since $t_{1}>0$, there is no agent who chooses to stay in state 1 , i.e. $\lambda\left(\mu_{1}\right)=0$. Contradiction. There is either complete or no brain drain, that is $\lambda\left(\mu_{1}\right)=0$ or $\lambda\left(\mu_{1}\right) \in\left[\frac{\mu_{1}}{2}, \mu_{1}\right]$.

Theorem 1.4. Assume there is no brain drain. There is a level $\hat{a}$, such that for any a $>\hat{a}$, agents will choose to stay state 1 , and for any $a<\hat{a}$, they choose to leave. For $\hat{a}$, the agent is indifferent if $\frac{w_{s}^{1}(\hat{a})-t_{1}}{w_{s}^{2}(\hat{a})}=1$. The fraction of agents who leave post graduation is given by $1-\lambda(\bar{a}, \hat{a})=\int_{\hat{a}}^{\bar{a}} f(z) d z$. (In the case of uniform distribution, $1-\lambda(\hat{a}, \bar{a})=(\hat{a}-\bar{a})$, and the fraction who stays by $\lambda(\hat{a}, \bar{a})=$ $(1-\hat{a})$ with $\hat{a} \in(0,1]$. 
Proof. We know that less than half leave state 1 , that is $\lambda(\bar{a})>\frac{\mu_{1}}{2}$. Assume $\lambda\left(\mu_{1}\right)<\mu_{1}$, otherwise the conclusion is trivial. Then $\lambda\left(\mu_{1}\right)>\mu_{1}-\lambda\left(\mu_{1}\right)$ and $g\left(\lambda\left(\mu_{1}\right)\right)-g\left(\mu_{1}-\lambda\left(\mu_{1}\right)\right)>0$. Let $a^{*}$ represent an agent who stays, i.e. $(g(\lambda(\mu))-g(\mu-\lambda(\mu))) a^{*}>t_{1}$. For any $a>a^{*}, g\left(\lambda\left(\mu_{1}\right)\right) a-g\left(\mu_{1}-\lambda\left(\mu_{1}\right)\right) a>$ $g\left(\lambda\left(\mu_{1}\right)\right) a^{*}-g\left(\mu_{1}-\lambda\left(\mu_{1}\right)\right) a^{*}>t_{1}$, and $a$ chooses to stay in state 1 . Let $a^{* *}$ an agent who leaves, i.e. $\left(g\left(\lambda\left(\mu_{1}\right)\right)-g\left(\mu_{1}-\lambda\left(\mu_{1}\right)\right)\right) a^{* *}<t_{1}$. For any $a<a^{* *}, g\left(\lambda\left(\mu_{1}\right)\right) a-g\left(\mu_{1}-\lambda\left(\mu_{1}\right)\right) a<$ $g\left(\lambda\left(\mu_{1}\right)\right) a^{* *}-g\left(\mu_{1}-\lambda\left(\mu_{1}\right)\right) a^{* *}<t_{1}$, and $a$ chooses to leave state 1 . Hence there is a $\hat{a} \in\left[\bar{a}, \frac{1-\bar{a}}{2}\right]$ such that for any $a>\hat{a}$, agents will choose to stay in state 1 , and for any $a<\hat{a}$, they choose to leave. From the continuity of $g$, it follows that for $\hat{a}$, the agent is indifferent if $\frac{w_{s}^{1}(\hat{a})-t_{1}}{w_{s}^{2}(\hat{a})}=1$.

\section{B Heterogeneity in returns to scale in higher education}

Proposition 1.5. Consider the environment where no subsidy is provided. There is a threshold $\bar{a}_{D R S}$ such that all individuals with ability $a>\bar{a}_{D R S}$ will enroll in college and all individuals with ability $a<\bar{a}_{D R S}$ will not enroll in college.

Proof. In the case $g(0)-c<1$, the highest ability agent, $a=1$, does not go to college. This implies that $\forall a$, if $g(0) a-c<1$, no one goes to college, i.e. $\bar{a}_{D R S}=1$.

In the case where $g(0)-c>1$, let $G(a)=g(\mu(a)) a-c$ with $G(0)=-c$ and $G(1)=g(0)-c$. Hence $G(0)<1<G(1)$, so by the Intermediate Value Theorem, there exists $\bar{a}_{D R S} \in(0,1)$ such that $g(0) \bar{a}_{D R S}-c=1$. In addition, since $\mu(a)=\int_{a}^{1} f(z) d z$ is continuous and decreasing in $a$, and $g^{\prime}(\mu)<0, G(a)$ is strictly increasing in $a$, the threshold $\bar{a}_{D R S}$ is unique and the result follows.

Proposition 1.6. Regardless of the subsidy type, a higher fraction $\mu_{D R S}$, will choose to go to school in state $1, \mu_{D R S}=\mu\left(\bar{a}_{1}\right)>\mu_{2}=\mu\left(\bar{a}_{2}\right)$. The fraction $\mu_{D R S}$, increases with the subsidy.

Proof. The result follows from Propositions 1.5 and 1.2.

Proposition 1.7. Given $\mu_{1}=\mu\left(\bar{a}_{1}\right)$, the fraction who enroll in college in state 1 , the fraction of agents who stay is $\lambda\left(\mu_{1}\right) \in\left[0, \frac{\mu_{1}}{2}\right)$. 
Proof. Suppose, by contradiction, less than half of college graduates leave state 1 , i.e. $\lambda\left(\mu_{1}\right) \geq \frac{\mu_{1}}{2}$. Then $\lambda\left(\mu_{1}\right)>\mu_{1}-\lambda\left(\mu_{1}\right)$, and since $g$ is decreasing, it follows that $g\left(\lambda\left(\mu_{1}\right)\right)-g\left(\mu_{1}-\lambda\left(\mu_{1}\right)<0\right.$. Then for any $a, g\left(\lambda\left(\mu_{1}\right)\right) a-g\left(\mu_{1}-\lambda\left(\mu_{1}\right)\right) a<t_{1}$. Hence agent $a$ chooses to leave state 1 . Contradiction. There is either complete or incomplete brain drain, that is $\lambda\left(\mu_{1}\right) \in\left[0, \frac{\mu_{1}}{2}\right)$.

Theorem 1.8. There is a level $\hat{a}$, such that for any $a>\hat{a}$, agents will choose to stay in state 1, and for any $a<\hat{a}$, they choose to leave. For $\hat{a}$, the agent is indifferent as $\frac{w_{s}^{1}(\hat{a})-t_{1}}{w_{s}^{2}(\hat{a})}=1$. The fraction of agents who leave post graduation is given by $1-\lambda\left(\bar{a}_{1}, \hat{a}\right)=\int_{\hat{a}}^{\bar{a}_{1}} f(z) d z$. (In the case of a uniform distribution, $1-\lambda\left(\hat{a}, \bar{a}_{1}\right)=\left(\hat{a}-\bar{a}_{1}\right)$, and the fraction who stays by $\lambda\left(\hat{a}, \bar{a}_{1}\right)=(1-\hat{a})$ with $\left.\hat{a} \in\left(\frac{1-\bar{a}}{2}, 1\right]\right)$.

Proof. We know that more than half of college graduates leave state 1 , that is $\lambda\left(\mu_{1}\right)<\frac{\mu_{1}}{2}$. Assume $\lambda\left(\mu_{1}\right)>0$, otherwise the conclusion is trivial. Then $\lambda(\mu)<\mu-\lambda(\mu)$ and $g(\lambda(\mu))-g(\mu-\lambda(\mu)>0$. Let $a^{*}$ represent an agent who stays, i.e. $(g(\lambda(\mu))-g(\mu-\lambda(\mu))) a^{*}>t_{1}$. For any $a>a^{*}$, $g(\lambda(\mu)) a-g(\mu-\lambda(\mu)) a>g(\lambda(\mu)) a^{*}-g(\mu-\lambda(\mu)) a^{*}>t_{1}$, and $a$ chooses to stay in state 1. Let $a^{* *}$ represent an agent who leaves, i.e. $(g(\lambda(\mu))-g(\mu-\lambda(\mu))) a^{* *}<t_{1}$. For any $a<a^{* *}$, $g(\lambda(\mu)) a-g(\mu-\lambda(\mu)) a<g(\lambda(\mu)) a^{* *}-g(\mu-\lambda(\mu)) a^{* *}<t_{1}, a$ chooses to leave state 1 . Hence there is a $\hat{a} \in(0,1]$ such that for any $a>\hat{a}$, agents will choose to stay in state 1 and for any $a<\hat{a}$, they choose to leave. From the continuity of $g$, it follows that for $\hat{a}$, the agent is indifferent if $\frac{w_{s}^{H}(\hat{a})-t_{1}}{w_{s}^{O}(\hat{a})}=1$. 